\title{
Updated Review of the Acoustic Modulation of Sleep: Current Perspectives and Emerging Concepts
}

\author{
Maren Jasmin Cordi $\mathbb{D}^{1,2}$ \\ 'Department of Psychology, Division of \\ Cognitive Biopsychology and Methods, \\ University of Fribourg, Fribourg, \\ Switzerland; ${ }^{2}$ Centre of Competence \\ Sleep \& Health Zurich, University of \\ Zurich, Zurich, Switzerland
}

\begin{abstract}
With growing interest in the use of acoustic stimuli in sleep research and acoustic interventions used therapeutically for sleep enhancement, there is a need for an overview of the current lines of research. This paper summarizes the various ways to use acoustic input before sleep or stimulation during sleep. It thereby focuses on the respective methodological requirements, advantages, disadvantages, potentials and difficulties of acoustic sleep modulation. It highlights differences in subjective and objective outcome measures, immediate and whole night effects and short versus long term effects. This recognizes the fact that not all outcome parameters are relevant in every research field. The same applies to conclusions drawn from other outcome dimensions, consideration of mediating factors, levels of stimulation processing and the impact of inter-individual differences. In addition to the deliberate influences of acoustic input on sleep, one paragraph describes adverse environmental acoustic influences. Finally, the possibilities for clinical and basic research-related applications are discussed, and emerging opportunities are presented. This overview is not a systematic review but aims to present the current perspective and hence summarizes the most up-todate research results and reviews. This is the first review providing a summary of the broad spectrum of possibilities to acoustically influence sleep.
\end{abstract}

Keywords: acoustic stimulation, sleep modulation, sleep disturbance, therapeutic intervention

\section{Introduction}

Since we are aware that we are less disconnected from the outside world during sleep than previously assumed, researchers are keen to find a bridge to the sleeping brain. ${ }^{1}$ Observations that auditory stimuli evoke electroencephalographic (EEG) responses in a sleeping brain ${ }^{1,2}$ have fueled investigations of acoustic modulations of sleep. One branch of research has continued to focus on stimulation during sleep. It mostly aims to find ways to promote sleep, ${ }^{3}$ examine consequences of disturbed sleep, mimic sleep disruptions ${ }^{4}$ or implement or foster cognitive processes during sleep. ${ }^{5}$ Acoustic stimulation is thus used as a means to experimentally manipulate specific sleep features to uncover their contribution to cognition, memory or health factors. Additionally, its impairing effects on sleep, for instance caused by nocturnal environmental noise, are in the scope of research (for an overview see ${ }^{6}$ ). Another line of research uses acoustic stimulation before sleep to explore its effects on the upcoming sleep period. These studies are mostly interested in clinical applications and aim to improve sleep or treat sleep disturbances, as will be outlined in the first
Correspondence: Maren Jasmin Cord University of Fribourg, Division of Cognitive Biopsychology and Methods, Rue P.A. de Faucigny 2, Fribourg, 1700, Switzerland

Tel +4I 263007678

Email maren.cordi@unifr.ch 
part of this review. As disrupted sleep is a risk factor for psychiatric $^{7}$ and a host of somatic illnesses ${ }^{8}$ and usually accompanies daytime impairments and increased risk for accidents, finding a side-effect free, easy to administer and inexpensive intervention is worthwhile.

This review tries to describe the current perspective on auditory stimulation both before and during sleep and outline their respective possible applications, new perspectives, goals and limits. This article offers a recent overview on the findings of the last three years whenever there was research available in this period. Else, earlier findings and historically relevant publications are also reported.

\section{Pre-Sleep Acoustic Modulation Evidence on the Subjective Level}

Presleep worrying and rumination not only disturb sleep directly ${ }^{9}$ but also mediate the relationship between stress and sleep even more strongly than somatic stress does. ${ }^{10}$ Conversely, people suffering from reduced sleep quality can benefit from a reduction in pre-sleep cognitive arousal. Intuitively, bedtime stories for children are among the most commonly used strategies of parents to promote their children's sleep. ${ }^{11}$ In an online survey, $62 \%$ of the respondents reported using music to improve their sleep. ${ }^{12}$ This preference to use music as a self-help strategy for sleep is related to its perceived efficacy. ${ }^{13}$ The latest systematic review and meta-analysis conducted in 2018 concluded that listening to music before bedtime was among the most powerful interventions for primary insomnia, as reflected by scores on the Pittsburgh Sleep Quality Index (PSQI) ${ }^{14}$ scores and sleep onset latencies. ${ }^{15}$ Among those studies, one trial showed that simply listening to soft, slow instrumental music regularly at any time of the day for 6 weeks reduced older adults' PSQI scores. ${ }^{16}$ Participants could choose between a variety of pieces of various genres that had relaxation potential and were of $60-80$ beats per minute (bpm) for a duration of 40 minutes. A more recent study replicated improvements in PSQI scores in healthy students. In a between-subjects design, the group that listened to the "tune Hejaz" (p. 24), supposed to be soothing and tranquilizing, for one hour per day, judged their sleep to be better at post measurement than the control group. ${ }^{17}$ Participants of a three-week music intervention versus listening to an audiobook of low emotional intensity (novels, fairy tales, short stories or magical realism) showed significant decreases in the insomnia severity index compared to a wait-list control group. ${ }^{18}$ In this trial, subjects could choose between classical, jazz, newage and ambient instrumental music of 50-80 bpm with a simple structure and stable dynamics, as those characteristics were assumed to be optimal for relaxation. Our research group experimentally tested, within subjects, the effects of a relaxing piece of music versus listening to a control text right before falling asleep. The music had specifically been composed to promote sleep using auditory pulsing of $0.25-2 \mathrm{~Hz}$ frequencies and judged to be one of the most relaxing ones out of 5 pre-selected pieces we had tested in a pilot study $\left(\mathrm{see}^{19}\right)$. In a commonly used sleep quality questionnaire ("Schlaffragebogen", SF-A ${ }^{20}$ ), subjects reported better sleep in the nap after this music. Contrary to all these positive effects, a very recent study showed that PSQI scores are negatively affected by the occurrence of earworms while falling asleep and awakening at night or in the morning. ${ }^{21}$ Furthermore, such involuntary music representation at sleep-related timepoints occurred more often in subjects who were highly engaged in music listening. This is the first study to demonstrate that music habits affect at what time earworms occur and that sleep-related habits are negatively associated with sleep quality.

While all previous studies tested healthy subjects, sleep improvements after music intervention could also be evidenced in patients suffering from fibromyalgia, ${ }^{22}$ after cardiac surgery in adults ${ }^{23}$ and adolescents ${ }^{24}$ and in patients with insomnia symptoms. ${ }^{25}$ The interventions were "delta-embedded" (p. 100) $2 \mathrm{~Hz}$ binaural beats on individual volume levels, ${ }^{22}$ soft and soothing music including pop music, opera, folk songs and light music for 30 minutes $^{24}$ or Wholetones ${ }^{\circledR}$ 2Sleep. ${ }^{25}$ The latter is tuned and layered with a particular frequency to lull into a deep sleep. Even outside the home setting, hospitalized children reduced subjective sleep disturbances with storytelling and music listening compared to a control group. ${ }^{26}$ Another study performed in the intensive care unit with postoperative older adults compared a standard care control group and a group listening to classical or contemporary instrumental music at bedtime for 30 minutes to a group that additionally had active music therapy during the day. ${ }^{27}$ This active group improved subjective sleep quality significantly more than the other groups. The difference between those was, however, not reported; hence, the contribution of acoustic stimulation before sleep per se cannot be evaluated.

Taken together, this line of research offers promising results demonstrating the effectiveness of music as a non- 
pharmacological intervention for sleep. In the majority of studies, no risks or side effects were reported, and music is usually superior to any other control condition (but $\sec ^{21,28}$ ). Usually, classical or instrumental music that had been judged to be relaxing or soothing was used. Such study results can diversify the field of sleeppromoting behaviour or even of evidence-based treatments of sleep disturbances. As some diagnostic criteria for sleep disturbances, for instance insomnia, are based on patients' sleep reports, positive effects of interventions on a subjective level are actually already conclusive. From a scientific perspective, however, objective correlates matter.

\section{Evidence on the Objective Level}

Currently, there is some promising evidence that the influence of music at bedtime is also measurable on an objective level. In addition to increased subjective sleep quality after using sedating music during the first hour in bed in young adults, the amount of slow-wave sleep (SWS) also increased after the intervention. ${ }^{29}$ Music therapists had especially composed sedative piano music of $60 \mathrm{bpm}$ in a "soft Asian style" (p. 313). The effect particularly appeared in subjects with long sleep latencies. This finding could hint at a particular benefit for sleep-disturbed patients. In contrast, another study investigating 15 subjects suffering from long sleep onset latencies did not report benefits from a $432 \mathrm{~Hz}$ music piece played 15-20 minutes before a nap at a comfortable volume. ${ }^{30}$ The frequency was chosen to be particularly calming, but contrary to what was expected, alpha power increased at sleep onset compared to non-music. A current systematic review investigated studies on complementary and alternative medical therapies for sleep in adult intensive care patients. ${ }^{31}$ Out of 360 initially identified studies, 17 were included. Four of them investigated music as therapy, and only one of them measured sleep polysomnographically. This study reported prolonged SWS and subjective sleep quality in the intervention group. ${ }^{32}$ According to previous characteristics of investigated music interventions, the authors had composed sedating piano music of 45 minutes length and a tempo of $60-80 \mathrm{bpm}$. The music had minor tonalities and refrained from dramatic changes in volume or rhythm to achieve a smooth melody line. The scarce availability of evidence for a modulating influence of music on objective sleep outcomes in patients might contribute to the relative neglect of pre-sleep acoustic interventions in the therapeutic daily routine.
However, evidence of beneficial effects of pre-sleep acoustic modulation on objective sleep parameters in healthy subjects is available. Using a hypnotic story played while falling asleep, we effectively and specifically increased young and older adults' amount of deep sleep in the following nap ${ }^{33,34}$ and nocturnal sleep. ${ }^{35}$ The story included a metaphor of a fish swimming deeper and deeper into the sea, suggesting the listener to let go and dive deeply into sleep. It followed a short hypnotherapeutic technique used to establish a calm, hypnotic state and was spoken in a slow, calming voice. In subjects not easily responding to hypnosis, the outcome on sleep was systematically reversed. ${ }^{36}$ Instead of an increase, they showed reductions in slow-wave sleep amounts. Even when replacing the hypnotic induction procedure with a breathing relaxation to prevent any possible arousing associations with hypnosis, this reverse effect was significant. ${ }^{36}$ This was not only contrary to our aim to improve sleep but also demonstrates that obviously unintended detrimental effects on sleep can appear. Whether the control text had increased deep sleep or the guided imagery decreased deep sleep could not be answered, as a neutral control group for a baseline measurement was lacking. In any case, it shows that pre-sleep acoustic interventions indeed have the power to manipulate the subsequent sleep architecture. Most likely, it acts through the guidance of thoughts on the arousal state or mood. A similarly unintended effect was shown in a less well-controlled but long term application in home setting. Gao et $\mathrm{al}^{37}$ asked subjects to listen to music or white noise for 20 minutes before bedtime for 6 days. For the sound intervention, the authors translated rapid eye movement (REM)- and SWS-related brain-wave characteristics into music pieces (eg brainwave amplitude was transposed to pitch). SWS-music significantly decreased sleep latency. While power in the delta band increased in the REMmusic and white noise groups, it decreased in the SWSmusic group. The authors interpreted this finding as the result of good sleep quality, leading to decreased sleep pressure. In contrast, a later commentary on this article questioned this conclusion. ${ }^{38}$ Again, the data proved that listening to a 20-minute piece of music before bedtime systematically influenced later sleep patterns but also showed unwanted directions of this alteration. Similarly, unexpected results were also objectively demonstrated in a study investigating sleep-related earworms. ${ }^{21}$ Polysomnographic measures showed worse sleep efficiency and sleep initiation after the instrumental versus the lyrical version of the same popular song. The authors assume that 
this was associated with the increased susceptibility to earworms at bedtime when instrumental music was played. Another study compared relaxing music during 30 minutes before going to bed to the effects of breathing exercise in young healthy adults. This instrumental sound was ambient, calm and slow, taken from the album "Sleep" by Max Richter. Significant reductions in N2 sleep and a trend towards increased slow-wave sleep were recorded across a whole night. ${ }^{39}$ This difference was, however, not reflected in changes in PSQI scores. The small sample size of this pilot study has to be taken into account when interpreting the results. In our study using a relaxing piece of music during falling asleep reported before, the subjective effects were also objectively measurable. In the nap after the intervention compared to the nap pattern after a control condition, we found reduced amounts of N1. ${ }^{19}$ To compare this effect to the hypnotic interventions we investigated in previous studies, we analysed subsamples of subjects scoring high versus low on hypnotizability. In contrast to reactions to the hypnotic story, subjects of low hypnotizability showed significant increases in SWS amounts and changes in power frequency bands after music intervention, indicating a shift towards less high frequencies and more low frequencies.

Which mechanism could be involved?

One explanation for how sound or music before sleep affects later sleep could be that it acts through mediating or moderating factors such as arousal reductions. ${ }^{32}$ Whether the intervention is capable of achieving a calming effect could, however, further depend on interindividual differences, such as health, relaxation abilities, or personal preferences. While in some subjects, only direct and active redirection of pre-sleep thoughts towards a sleep-promoting path reduces arousal, others might benefit from the possibility of disconnecting from the outside world during unstructured music listening. Therefore, the sort of condition the acoustic intervention was tested against must be considered. Apart from one study lacking a control group, ${ }^{22}$ studies compared music listening to a diversity of controls. Among them were nonintervention controls, ${ }^{17,30}$ comparisons to other music pieces, ${ }^{25,40}$ other auditory inputs such as audiobooks, ${ }^{19,26,41}$ rest or quiet periods ${ }^{16,23,24}$ or a combination of any other auditory stimulation and nonintervention. ${ }^{26,27,41}$ All of them found beneficial effects on subjective sleep quality ratings even though the intervention duration ranged from a single 20 -minute listening ${ }^{19}$ to 30 minutes per day across 6 months. ${ }^{24}$ Studies measuring objective sleep parameters mainly included comparisons of the acoustic intervention to measures after falling asleep without music $29,30,32$ or to listening to other versions of the music ${ }^{21}$ or other texts. ${ }^{19,33-36}$ One other study compared two pieces of music to white noise, ${ }^{37}$ and another study compared it to breathing relaxation. ${ }^{39}$ In a majority, the intervention took place for 15 to 45 minutes before falling

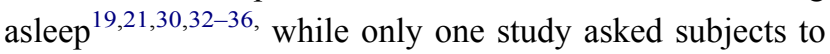
listen to the music for 20 minutes each day for 6 days. ${ }^{37}$ Apart from control conditions and exposure time, research has shifted attention towards the potential influence of certain frequencies. Although $432 \mathrm{~Hz}$ music pieces are considered particularly calming, no objective differences to a non-intervention control were found. ${ }^{30}$ Again, sleep was still subjectively rated better after a $432 \mathrm{~Hz}$ version of a music piece compared to tuning it to $440 \mathrm{~Hz}^{40}$ Even effects of a particularly "composed" music, created by translating EEG signals into tone characteristics (eg, EEG amplitude into pitch, power to volume, etc.) on sleep were tested. Nevertheless, neither SWS-wave music nor REM-wave music influenced sleep structure, although the former reduced sleep onset latency and delta power. ${ }^{37}$ One study using binaural beats did not include a control group; thus, placebo effects or changes due to mere passage of time cannot be excluded. ${ }^{22}$ The other one compared the same piece of music the subjects listened to across two weeks with and without binaural beat stimulation. This manipulation, however, did not augment the subjectively rated benefits. ${ }^{42}$ The same conclusion was drawn when comparing Wholetones ${ }^{\circledR}$ 2Sleep, using "frequency-enhanced music and precise tempos" 25 to classical music. PSQI scores improved equally after both acoustic interventions. Other sound specifications, such as volume and tempo, are reported very scarcely and have not been systematically tested. If it was indicated at all in the papers, it was tuned to $42-50 \mathrm{~dB}^{19,21}$ or self-chosen by the subjects and hence not controlled ${ }^{24,39}$ and approximately 50 to $80 \mathrm{bpm}^{18}$

In sum, it remains unclear which frequency or special effect of sound benefits sleep most. That is, efforts to bring frequencies to perfection have been inconclusive thus far. However, findings must be differentiated between subjective and objective effects. Objective results were much more inconsistent than subjective ratings, as zero findings and even reverse effects (reduced deep sleep, sleep efficiency or delta power) were measured. Obviously, subjective ratings are more robust to the advantage of music as a method for relaxation, outperforming all other 
possibilities to calm down. Sounds may also simply act more directly on emotions and mood than other relaxation techniques. This leads to the idea that personal music preferences or cultural influences play a decisive role. While some subjects enjoy slow, peaceful, relaxing music and can calm down during listening, others might be annoyed by it. Indeed, due to experimental reasons, most studies used music that was considered "relaxing", "soothing", "soft", “calm", “ambient", "slow" or "sedative"24,29,39, and applied the same piece to each subject. What is considered relaxing, soft, soothing or sedative was tested in a pilot study, ${ }^{19}$ derived from prior studies $^{18,27}$ or defined by the authors, ${ }^{24,39}$ music therapists $^{29}$ or the "NIH music recommendations" 25 (p.303) and mostly resulted in classical music pieces or instrumental music. Only a few subjects were allowed to choose from a small pool of music pieces. ${ }^{18,24}$ Those were then, however, preselected as well and out of categories such as "light music, folk songs, opera and pop music" 24 or "classical, jazz, new-age and ambient"18 (p.3). Whether any kind of personal music preference has an influence on the efficacy of the intervention was investigated in a group of Japanese students. Yamasato and colleagues ${ }^{43}$ found that prescribed sedative music as well as any type of preferred piece of music during bedtime every night for 4 weeks reduced PSQI scores compared to a no-music control group. While global PSQI scores did not differ between prescribed versus chosen music, differences in some of the subscores appeared. In general, only the scores of the given sedative music fell below the clinical cut-off value of 6 points. Taken together, music seems to influence sleep even if the piece itself does not match personal music preferences.

In summary, large-scale, randomized controlled clinical studies demonstrating effectiveness, safety and longterm effects, especially on the objective level, particularly in patients, are scarce. The studies available show systematic effects on sleep, which are, however, not always to the advantage of sleep quality. Whether it is any characteristic of auditory input that is crucial for the consequences for sleep or if mediators come into play that must be identified is still unclear. In contrast, studies in healthy subjects and those including subjective sleep improvements are quite in agreement about its potential, and intuitively, music is frequently used as a therapeutic tool. As a limiting factor, it must be considered that, as a consequence of the file drawer problem, zero findings or negative study results have likely not been published.
Moreover, methodological problems and biases (eg, it is impossible to blind the conditions) can influence subjective reports or provoke expectancy effects. Another limitation is that inter-individual differences in the effect of sounds and stories (ie, hypnotic suggestions, guided imagery) on sleep appeared, while the reasons have thus far not been uncovered. Identifying such potential mediators would be highly relevant to develop a theoretical framework that can be tested systematically. Moreover, it has been criticized that studies thus far tested standardized music treatments on standardized outcomes, disrespecting patient customization for best care practices. ${ }^{44}$ Such an approach might mask the interventions' potential. The mixed results leave clinical workers with many uncertainties, making them reluctant to implement music as a therapeutic tool in their clinical applications. However, as it does not need investments in special trainings or material, it is indeed an attractive tool for healthcare professional institutions. To establish its application in clinical settings, it might also be necessary to transfer this knowledge in schools or clinical education programs to increase awareness of music as a treatment.

\section{Acoustic Stimulation During Sleep Sleep Improvements}

During sleep, information processing and a vast number of other cognitive and physiological aspects change. Inhibition of incoming signals relates to the loss of consciousness when falling asleep. Most likely, the reticular activating system (RAS), critically involved in sleep-wake transition and arousals during sleep, plays a major role in this process. ${ }^{45}$ While external stimuli are supposed to be blocked in their thalamic-cortical path, ${ }^{46}$ event-related potentials can still be quantified when input is processed. ${ }^{47}$ Whether the sleeper only shows an EEG response, an arousal, shifts into a lighter sleep stage or awakens depends on the sleep stage and several criteria of the stimulus presented. ${ }^{48}$ The literature suggests that such sound-related arousals or changes could be a consequence of a sympathetic response or follow a modulation in ion channels. ${ }^{6}$ They could represent the underlying mechanism of k-complexes, which can be elicited by acoustic stimuli such as tones. ${ }^{49}$ Their similarity to slow oscillations (SO), which are supposed to be critically involved in memory consolidation, has provoked researchers to manipulate their occurrence with acoustic stimulation. Recently, Choi et $\mathrm{al}^{50}$ reviewed 148 studies targeting the 
manipulation of sleep, among other targets, by acoustic stimulation. In open-loop paradigms, auditory stimulation is considered independent of the brain state. By comparing sleep patterns in the stimulation versus control condition, it might be demonstrated that specific sleep parameters can indeed be manipulated by acoustic stimulation. For instance, presenting rhythmic pink noise bursts in sleep stage N2 increased slow oscillation power in NREM and slow-wave sleep during the stimulation period and 10-25 minutes later. The sleep stage distribution in general was not affected, neither during nor after the stimulation. ${ }^{51}$ This study demonstrated for the first time that this approach can be used to investigate sleep functions. One important conclusion, however, was that this effect critically depends on the prevailing brain state during presentation. An optimization of this method was thus the closedloop paradigm, which detects brain states online for a more precise timing of the stimulation. A feedback tool controls the effect of the exhibited manipulation on neural activity. Therefore, it can influence the investigated parameters, such as slow oscillations or sleep spindles, more systematically. Such precise, selective and exclusive stimulation of certain sleep parameters is required to unravel sleep mechanisms. Moreover, this approach increases controllability and includes a stimulation protocol that can be reproduced and replicated. This enabled researchers to include behavioural effects beyond mere sleep manipulation in the measures. In a follow-up study of the same group of researchers, introducing pink noise precisely at the upstate of the slow wave, not only replicated the enhancement of the SO rhythm but also led to an improvement in declarative memory consolidation. ${ }^{52,53}$ Again, the effect was restricted to the oscillation and did not affect general sleep architecture. Others have confirmed changes in slow-wave characteristics as well as a memory benefit due to stimulation with pink noise on the slow oscillation in younger ${ }^{54}$ and older adults. ${ }^{55}$ Additionally, targeting sleep spindle activity with auditory stimuli can be implemented and has shown a beneficial effect on procedural memory consolidation. ${ }^{56}$ The authors, however, highlighted the importance of the sound level of the stimuli as well as their timing for the observation of the effects. As a variety of these determinants can influence the outcome, it is not surprising that the results are mixed and that other studies did not find behavioural effects, for instance, in a spatial navigation task after stimulation, despite changes in slow oscillations and spindle activity being confirmed. ${ }^{57}$ Apart from deepening NREM sleep and supporting memory formation, auditory closed-loop stimulation of SO intensified the expression of endocrine measures that are involved in immune functions. ${ }^{58}$ Thus, consequences at the whole body level are prevalent. Furthermore, presenting phase-locked tones during SWS on two consecutive nights increased slow-wave activity (SWA) without changing sleep architecture compared to sham stimulation with inaudible tones. ${ }^{59}$ In the second line, this was followed by increased subjective feelings of alertness and objective performance in attentional tasks on both next days. Previously, subjects selfrestricted their sleep schedule to simulate chronic insufficient sleep and its consequences for cognition. The results showed that acoustic stimulation could alleviate some cognitive deficits associated with sleep restriction. All previously mentioned studies investigated single laboratory nights. An observational pilot study tested wearable devices to implement usage in the home environment and to allow assessment of 10 consecutive nights of stimulation. The increase in the slow oscillation response shortly after stimulation remained comparable to the first night measured in the laboratory across all nights in the home environment. ${ }^{60}$ This hints at a lack of habituation to acoustic stimulation, which would imply that its efficacy does not diminish over time. Although promising and worth pursuing, a recent review on slow-wave sleep manipulation through brain stimulation concluded that there is currently not sufficient evidence for the effectiveness of such devices. ${ }^{61}$

Similar to the results of acoustic stimulation before sleep, huge inter-individual variability in the effectiveness of SWA stimulation has been observed. Possible traits or individual factors related to the extent of the response to acoustic stimulation in terms of slow-wave enhancement are, again, not yet discovered. Age has already been discussed as one of these factors. In a middle-aged group, the effects of the same auditory stimulation protocol used for younger adults on SO and sleep spindles were markedly diminished and differed in their temporal pattern. This also affected the degree of declarative memory retention. ${ }^{62}$ In addition to age, other conditions altering sleep composition, such as psychiatric illness, can modulate the influenceability of sleep via acoustic stimulation. Moreover, it is important to emphasize again that all those effects referred to specific manipulations restricted to the stimulation period or even the few seconds immediately after the stimulation. Although most of the studies reported no changes in overall sleep structure, not all of them paid attention to the 
unstimulated part of sleep, neglecting possible detrimental consequences. Therefore, the upcoming paragraph focuses on the rest of the sleep episode.

\section{Immediate Versus Whole Night Effects}

Generally, the results can vary depending on whether immediate effects of acoustic stimulation on sleep are considered or if changes across the whole night are taken into account. Schade and colleagues ${ }^{63}$ recently tested nonphase-locked pink noise to enhance the characteristics of deep sleep against environmental sounds presented throughout sleep to disrupt those features in a withinsubjects design. Compared to sham stimulation, both acoustic stimuli increased delta and slow-oscillatory power during and close to stimulation. Across the whole night, however, disruptions diminished power in those frequency bands, such as the duration and proportion of SWS and sleep continuity. This was followed by impaired next-day valiance performance. ${ }^{63}$ In contrast, the enhancing condition preserved delta and SO activity as well as sleep efficiency and quality across the night compared to the sham condition. Thus, despite similar immediate beneficial effects of stimulation in both conditions, their overall effect on the whole sleep period differed dramatically and was followed by very divergent consequences on a behavioural level. Especially if secondary outcomes such as next-day performance or memory are in the scope of investigation, this study demonstrated how important the inclusion of both perspectives, the immediate effects and changes in the entire sleep episode, is. Fostering the importance of this differentiation, it was discussed that the on- and offset of noise is particularly harmful for sleep and health. ${ }^{64}$ Consequently, a systematic review summarized the effects of continuous noise on sleep during the entire sleep episode. ${ }^{65}$ The authors mainly focused on white noise, which was hypothesized to have the potential to be beneficial, as its nature has the potential to mask other disruptive sounds. Overall, the data showed that continuous noise is not harmful but indeed actually tended to be helpful for sleep initiation and continuation, even though evidence for such a beneficial effect was low.

\section{Beyond Physiological Reactions to Acoustic Stimuli}

An emerging new perspective has currently been tested experimentally for the first time. The brain has been demonstrated to be receptive to acoustic input at the physiological level, but consciousness or other higherlevel cognitive processes might be accessible as well. Methodologically, thus, the technique to acoustically present mere tones during sleep was further advanced by combining it with elements from targeted memory reactivation (TMR). This method originating from memory research aims to externally initiate reactivation of previously learned material during sleep. This replay was shown to promote consolidation of those memories. Usually, cues such as words or sounds, taken from or associated with the pre-sleep learning task, are used as acoustic triggers during sleep. It seems to act on firing rates of newly established synaptic connections, and it was hypothesized that whole schematic concepts could also be activated. Those schemas include a diversity of experience-based components such as body sensations and emotional tone as elements of the according memories. The hypothesis that relaxation-related words can activate the concept of relaxation when played during sleep was thus investigated recently in our lab. Fifty healthy subjects were presented relaxing words during NREM sleep. This resulted in increased time spent in SWS, more power in SWA and better subjective sleep quality the next morning. ${ }^{66}$ Thus, apart from a mere electrophysiological response to external stimulation, this study hinted at the existence of another level on which acoustic stimulation during sleep could act. If embodied concepts can indeed be activated directly without the need for conscious awareness, this has a highly relevant therapeutic potential. Similar to the basic concepts of hypnotherapeutic techniques, changes could be initiated circumventing blockage by a critical cognitive entity. As this was the first study to test this approach, further studies are of course needed to replicate the findings and extend our understanding.

Taken together, acoustic stimulation can be used to manipulate sleep characteristics quite precisely. Studies must be differentiated between immediate effects and whole-night effects. While immediate effects might be more relevant for basic research, the global changes in sleep are more relevant for clinical applications. To elucidate the functions of certain sleep characteristics, it is relevant to increase the precision of the stimulation timing. Mere tones or noise can be instrumentalized to modulate single sleep parameters and result in benefits for cognition, memory, immune parameters and subjective and objective alertness. In addition to this rather technical side, interesting first hints towards a next level of processing during sleep could have been provided. Whole semantic concepts may 
be activated by presenting specific cue words during sleep. This indicates that the type of acoustic stimulation is also relevant for its effect on sleep and shows that there might be several levels on which acoustic stimuli can influence sleep: mere physiological reactions and higher-order processes. Pursuing this account could offer new therapeutic possibilities not only specifically for sleep disturbances but also general psychotherapy. Along with considerations of patient-centred interventions, inter-individual differences have been observed, which are still unclear. The reasons for how or to what extent acoustic stimulation before or during sleep affects sleep were discussed in relation to age, personality traits, sleep quality or psychiatric health.

\section{Undesired Effects of Acoustic Stimulation During Sleep}

While thus far only positive effects and deliberate acoustic stimulation during sleep have been considered, it must be mentioned that sound might also influence sleep in an undesirable way. This section does not claim to present the complete scientific background available on this topic, which is immense, but in this context, there is need to include the discussion on unwilling exposure to sounds. Thus, only the most recent findings about its physical and psychological consequences are outlined.

The effects of turbine noise or traffic noise are discussed as potential health-impairing factors. The WHO has defined the sound level inside bedrooms to $45 \mathrm{~dB}$, outside $60 \mathrm{~dB}$ as the lowest critical value able to produce adverse health effects, such as sleep disturbances. ${ }^{67}$ These levels refer to the general population and thus could be even lower for vulnerable groups or specific sound characteristics (low frequency, low background noise). ${ }^{68}$ These exceptions, together with a low number of methodologically sound studies and diverging study findings, result in uncertainty about those thresholds ${ }^{48}$ and the effects of specific sound characteristics. ${ }^{69}$ A recent systematic review and meta-analysis, for instance, could not confirm that wind turbine noise impairs objective sleep onset latency, total sleep time, sleep efficiency or wakefulness after sleep onset. ${ }^{70}$ Another review summarizes previous reviews and concludes that "well-conducted studies using objective measures of sleep and acoustics are scarce and highly warranted" 69 because otherwise, no firm causal influence of wind turbine noise on sleep disturbance can be concluded. Only since the last WHO release 34 new studies on sleep disturbance caused by environmental noise have been published up to December 2019, out of which three measured sleep objectively. ${ }^{71}$ From a clinical point of view, however, it is less clear that only objectively measurable sleep manipulation should be considered relevant. As some of the above-mentioned studies or reviews, such as Liebich et al${ }^{70}$, did not meta-analyse the subjective measures of sleep, it cannot be concluded that noise did not disturb sleep. Referring to a vast number of studies reporting diverging measures of objective and subjective sleep parameters in healthy and even more pronounced in sleep-disturbed patients, ${ }^{72,73}$ the possibility of subjective sleep disturbance is not unlikely even in the absence of objective changes. In particular, criteria for sleep disturbances, such as insomnia, are defined by subjective sleep reports and do not need an objective correlate to be clinically relevant. Moreover, even if sleep modulation is not measurable, or not to the same extent as subjective reports, its impact on wellbeing, daytime tiredness, and thereby risk for health is still a fact. Acoustic influences on sleep not only provide advantages, but the sensitivity to sounds during sleep also has inherent undesired side-effects.

Critically, in this regard, there is little evidence on the long-term effects of acoustic input on sleep. While habituation effects could be expected, limiting the impact of sounds with increasing duration of exposure, studies usually show small habituation effects, as reported in Micic et al. ${ }^{69}$ What was considered an advantage in the long term home study of SWA stimulation $^{60}$ may also have risks: increased heart rate and body movements persisted for two weeks of nocturnal traffic noise exposure, and no signs of habituation appeared for sleep quality, mood or performance. ${ }^{74}$ Eight consecutive nights of ship noise exposure showed no habituation in actigraphic measures but changes in some aspects of subjective sleep quality. ${ }^{75}$ The same pattern appeared across 10 nights of traffic noise, as reported by Kuroiwa et al.: ${ }^{76}$ while no evidence for habituation was found in polysomnographic measures, all subjective parameters showed adaptation. In another field study, sleep quality and mood-related measures were diminished in inhabitants of an area exposed to traffic noise compared to a control area, while tiredness and diverse somatic complaints were reported more often. ${ }^{77}$ Noise sensitivity, however, again emerged as important in these results and again raises the issue of inter-individual variability and the 
differentiation between objective and subjective sleep measures.

\section{Other Indirect or Nonspecific Effects of Acoustic Modulation on Sleep}

What might be related to the inter-individual findings, especially in subjective sleep reports, is the major role of mediating psychophysiological mechanisms. Frei et $\mathrm{al}^{78}$ demonstrated that annoyance about noise was the relevant mediating factor in the relationship between subjective sleep quality and noise, while actual noise exposure was not. At the same time, annoyance did not correlate with objective sleep as measured by actigraphy. Actigraphic measures were only dependent on real noise exposure. Whether sleep can be disrupted or manipulated hence depends not only on specific sound characteristics but also on the degree of annoyance. If subjective sleep disturbance is taken into account, this "secondary" pathway of acoustic sleep manipulation should also be considered relevant, as its physiological and psychological consequences still persist. In addition to annoyance as an additional, although indirect influence on sleep, general effects of noise on body perceptions other than hearing also exist, such as body vibration or pressure in the ear or head, which have been reported. ${ }^{79}$ Of course, such sensations can also impact sleep or interact with annoyance.

\section{Discussion}

In this review, the results of studies, meta-analyses and reviews on the effects of acoustic stimulation on sleep are outlined. While this enabled covering a broad spectrum of topics, it cannot offer a perfectly complete summary that a systematic review or meta-analysis could provide. Instead, it can highlight differences and commonalities of the research "branches" that have grown out of the investigation of acoustic stimuli and sleep, detect their advantages and needs and compare and relate their results.

In summary, it can be concluded that studies quite consistently show that acoustic stimulation before sleep can positively act on subjective sleep reports of healthy and sleep-disturbed subjects. Thus, therapeutic use could already be drawn from these observations. Investigations on objective outcomes are scarce, especially in clinical samples. In healthy subjects, some evidence is available, which indicates that additional influential factors come into play, which are not yet fully understood. Uncovering the underlying mechanisms of how and why music or other acoustic interventions improve sleep could offer possibilities to understand and make use of specific factors deciding over the success or failure of the intervention. Second, the literature outlining the current perspective on acoustic stimulation during sleep was discussed. Here, acoustic stimulation is used to advance the basic understanding of the brain state of sleep and its importance for secondary outcomes such as memory, health or daytime performance. As application with this intention targets concrete and isolated aspects of sleep, exact timing, sound characteristics and study designs are decisive. The results converge and demonstrate its effectiveness, especially for immediate impact. However, whole-night effects are less commonly under investigation and can greatly diverge from the latter. Finally, in addition to intentional stimulation, undesired nocturnal disturbances distort sleep quality in everyday life. This again has consequences on objective and subjective levels, while mediating factors play an important role in this relationship.

Overall, the results pattern generally demonstrated that effects on subjective sleep reports and objective sleep parameters must be differentiated. Their outcomes do not always correspond. Diverse reasons for this divergence could be assumed. Either certain acoustic stimuli indeed act only on certain processes or only parameters that are irrelevant to sleep perception have been targeted. However, it is still unclear which aspects of sleep are relevant for subjective sleep quality. ${ }^{80}$ However, even given "accurate" sleep perception, subjects' ratings might be influenced by mediating factors, such as annoyance, unblinded study designs or expectancy effects. This divergence in outcome measures has to be taken into account when designing studies.

Following this outline, the aim of the acoustic intervention should decide on timing, sound characteristics and presentation of the stimuli as well as the relevant outcome parameters. If, for instance, clinical applications in terms of patient care, prevention, therapeutic intervention or general healthcare in widespread populations are targeted, subjective outcomes might be pivotal. If basic mechanisms of sleep are investigated, objective outcomes are clearer. With therapeutic purposes in mind, the intervention can be provided before sleep or while falling asleep and needs to fulfill less strict requirements for the exact timing of sounds, which is, on the contrary, essential for basic research. Finally, meaningful words, instead of sounds, may operate at a level higher than the physiological. 


\section{Conclusion}

In general, acoustic stimuli offer diverse possibilities to influence sleep and can lead to desired but also undesired sleep manipulations. Depending on the aim of intentional acoustic stimulation, it must be well considered whether subjective or objective levels are relevant and targeted. Acoustic intervention offers great potential for therapeutic use as well as precise, side-effect-free manipulation of sleep parameters relevant to advance the field of sleep research. It is hence a tool that provides usability for basic research questions as well as potential as an applied therapeutic tool. While its use in research has already been acknowledged, its practical application still lags behind unnecessarily.

\section{Abbreviations}

EEG, electroencephalogram; NREM, non-rapid eye movement; PSQI, Pittsburgh Sleep Quality Index; REM, rapid eye movement; RAS, reticular activating system; SO, slow oscillations; SWA, slow-wave activity; SWS, slow-wave sleep; TMR, targeted memory reactivation.

\section{Funding}

This work has received funding from the European Research Council (ERC) under the European Horizon 2020 research and innovation program (Grant agreement No. ERC-2015-STG 677875).

\section{Disclosure}

Dr Maren Jasmin Cordi reports grants from European Research Council (ERC) in relation to the submitted work. The author reports no other conflicts of interest in this work.

\section{References}

1. Davis H, Davis PA, Loomis AL, Harvey EN, Hobart G. Electrical reactions of the human brain to auditory stimulation during sleep. J Neurophysiol. 1939;2(6):500-514. doi:10.1152/jn.1939.2.6.500

2. Keefe FB, Johnson LC, Hunter EJ. EEG and autonomic response pattern during waking and sleep stages. Psychophysiology. 1971;8 (2):198-212. doi:10.1111/j.1469-8986.1971.tb00451.x

3. Webb WB, Agnew HW. Sleep onset facilitation by tones. Sleep. 1979;1(3):281-286. doi:10.1093/sleep/1.3.281

4. Thiessen GJ, Lapointe AC. Effect of continuous traffic noise on percentage of deep sleep, waking, and sleep latency. J Acoust Soc Am. 1983;73(1):225-229. doi:10.1121/1.388853

5. Guerrien A, Dujardin K, Mandal O, Sockeel P, Leconte P. Enhancement of memory by auditory stimulation during postlearning REM sleep in humans. Physiol Behav. 1989;45(5):947-950. doi:10.1016/0031-9384(89)90219-9

6. Liu J, Ghastine L, Um P, Rovit E, Wu T. Environmental exposures and sleep outcomes: a review of evidence, potential mechanisms, and implications. Environ Res. 2020;110406. doi:10.1016/j. envres.2020.110406
7. Baglioni C, Battagliese G, Feige B, et al. Insomnia as a predictor of depression: a meta-analytic evaluation of longitudinal epidemiological studies. J Affect Disord. 2011;135(1-3):10-19. doi:10.1016/j. jad.2011.01.011

8. Cappuccio FP, Miller MA. Sleep and Cardio-Metabolic Disease. Vol. 1. Oxford University Press;2018. doi:10.1093/oso/97801987 78240.003.0008

9. Ackermann S, Cordi M, La Marca R, Seifritz E, Rasch B. Psychosocial Stress Before a Nap Increases Sleep Latency and Decreases Early Slow-Wave Activity. Front Psychol. 2019;10. doi:10.3389/fpsyg.2019.00020

10. Tousignant OH, Taylor ND, Suvak MK, Fireman GD. Effects of Rumination and Worry on Sleep. Behav Ther. 2019;50(3):558-570. doi:10.1016/j.beth.2018.09.005

11. Schlarb AA, Seiler D, Werner A, Kater MJ. Modern strategies for settling infants to sleep: a comparison between sleep-disturbed and non-sleep-disturbed infants. Somnologie. 2020;24(4):237-244. doi:10.1007/s11818-020-00279-0

12. Trahan T, Durrant SJ, Müllensiefen D, Williamson VJ. The music that helps people sleep and the reasons they believe it works: a mixed methods analysis of online survey reports. PLoS One. 2018;13(11): e0206531. doi:10.1371/journal.pone.0206531

13. Huang C-Y, Chang E-T, Lai H-L. Use of integrative medicine approaches for treating adults with sleep disturbances. Appl Nurs Res. 2018;43:49-55. doi:10.1016/J.APNR.2018.06.016

14. Buysse DJ, Reynolds CF, Monk TH, Berman SR, Kupfer DJ. The Pittsburgh Sleep Quality Index: a new instrument for psychiatric practice and research. Psychiatry Res. 1989;28(2):193-213. doi:10.1016/0165-1781(89)90047-4

15. Feng F, Zhang Y, Hou J, et al. Can music improve sleep quality in adults with primary insomnia? A systematic review and network meta-analysis. Int J Nurs Stud. 2018;77:189-196. doi:10.1016/j. ijnurstu.2017.10.011

16. Shum A, Taylor BJ, Thayala J, Chan MF. The effects of sedative music on sleep quality of older community-dwelling adults in singapore. Complement Ther Med. 2014;22(1):49-56. doi:10.1016/j. ctim.2013.11.003

17. Kavurmaci M, Dayapoğlu N, Tan M. Effect of music therapy on sleep quality. Altern Ther Health Med. 2020;26(4):22-26.

18. Jespersen KV, Otto M, Kringelbach M, Van Someren E, Vuust P. A randomized controlled trial of bedtime music for insomnia disorder. J Sleep Res. 2019;28(4):e12817. doi:10.1111/jsr.12817

19. Cordi MJ, Ackermann S, Rasch B. Effects of Relaxing Music on Healthy Sleep. Sci Rep. 2019;9(1):9079. doi:10.1038/s41598-01945608-y

20. Görtelmeyer R. SF-A/R Und SF-B/R - Schlaffragebogen A Und B Revidierte Fassung. Hogrefe; 2011.

21. Scullin MK, Gao C, Fillmore P. Bedtime Music, Involuntary Musical Imagery, and Sleep. Psychol Sci. 2021;095679762198972. doi:10.1177/0956797621989724

22. Picard LM, Bartel LR, Gordon AS, Cepo D, Wu Q, Pink LR. Music as a sleep aid in fibromyalgia. Pain Res Manag. 2014;19(2):97-101. doi:10.1155/2014/272108

23. Zhang QL, Xu N, Huang ST, et al. Music Therapy for Early Postoperative Pain, Anxiety, and Sleep in Patients after Mitral Valve Replacement. Thorac Cardiovasc Surg. 2020;68(6):498-502. doi: $10.1055 / \mathrm{s}-0040-1713352$

24. Lin ZW, Liu JF, Xie WP, Chen Q, Cao H. The effect of music therapy on chronic pain, quality of life and quality of sleep in adolescents after transthoracic occlusion of ventricular septal defect. Heart Surg Forum. 2021;24(2):E305-E310. doi:10.1532/hsf.3513

25. Hausenblas H, Hooper S, Hooper D, Coyle K, Lynch T. Efficacy of Wholetones ${ }^{\circledR}$ 2Sleep and classical music on sleep and health behaviors of adults with insomnia symptoms: a single blind, randomized, controlled, crossover pilot trial. Sleep Sci. 2019;12(4):302-306. doi:10.5935/1984-0063.20190091 
26. Anggerainy SW, Wanda D, Nurhaeni N. Music Therapy and Story Telling: nursing Interventions to Improve Sleep in Hospitalized Children. Compr Child Adolesc Nurs. 2019;42(sup1):82-89. doi:10.1080/24694193.2019.1578299

27. Kim J, Choi D, Yeo MS, Yoo GE, Kim SJ, Na S. Effects of patient-directed interactive music therapy on sleep quality in postoperative elderly patients: a randomized-controlled trial. Nat Sci Sleep. 2020;12:791-800. doi:10.2147/NSS.S286375

28. Lazic SE, Ogilvie RD. Lack of efficacy of music to improve sleep: a polysomnographic and quantitative EEG analysis. Int J Psychophysiol. 2007;63(3):232-239. doi:10.1016/j.ijpsycho.20 06.10 .004

29. Chen C-K, Pei Y-C, Chen N-H, et al. Sedative music facilitates deep sleep in young adults. J Altern Complement Med. 2014;20 (4):312-317. doi:10.1089/acm.2012.0050

30. Dubey P, Kumar Y, Singh R, Jha K, Kumar R. Effect of music of specific frequency upon the sleep architecture and electroencephalographic pattern of individuals with delayed sleep latency: a daytime nap study. J Fam Med Prim Care. 2019;8(12):3915. doi:10.4103/ jfmpc.jfmpc_575_19

31. Cooke M, Ritmala-Castrén M, Dwan T, Mitchell M. Effectiveness of complementary and alternative medicine interventions for sleep quality in adult intensive care patients: a systematic review. Int $J$ Nurs Stud. 2020;107:103582. doi:10.1016/j.ijnurstu.2020.103582

32. Su CP, Lai HL, Chang ET, Yiin LM, Perng SJ, Chen PW. A randomized controlled trial of the effects of listening to non-commercial music on quality of nocturnal sleep and relaxation indices in patients in medical intensive care unit. $J$ Adv Nurs. 2013;69 (6):1377-1389. doi:10.1111/j.1365-2648.2012.06130.x

33. Cordi MJ, Schlarb AA, Rasch B. Deepening sleep by hypnotic suggestion. Sleep. 2014;37(6):1143-1152. doi:10.5665/sleep.3778

34. Cordi MJ, Hirsiger S, Mérillat S, Rasch B. Improving sleep and cognition by hypnotic suggestion in the elderly. Neuropsychologia. 2015;69:(March):176-182. doi:10.1016/j.neuropsychologia.20 15.02.001

35. Cordi MJ, Rossier L, Rasch B. Hypnotic Suggestions Given Before Nighttime Sleep Extend Slow-Wave Sleep as Compared to a Control Text in Highly Hypnotizable Subjects. Int J Clin Exp Hypn. 2020;68 (1):105-129. doi:10.1080/00207144.2020.1687260

36. Cordi MJ, Rasch B. Systematic decrease of slow-wave sleep after a guided imagery designed to deepen sleep in low hypnotizable subjects. J Sleep Res. 2020. doi:10.1111/jsr.13168

37. Gao D, Long S, Yang H, et al. SWS Brain-Wave Music May Improve the Quality of Sleep: an EEG Study. Front Neurosci. 2020:14. doi:10.3389/fnins.2020.00067

38. Johnson JM, Durrant SJ. Commentary: SWS Brain-Wave Music May Improve the Quality of Sleep: an EEG Study. Front Neurosci. 2021;15:67. doi:10.3389/fnins.2021.609169

39. Kuula L, Halonen R, Kajanto K, et al. The Effects of Presleep Slow Breathing and Music Listening on Polysomnographic Sleep Measures - a pilot trial. Sci Rep. 2020;10(1):1-9. doi:10.1038/ s41598-020-64218-7

40. Calamassi D, Lucicesare A, Pomponi GP, Bambi S. Music tuned to $432 \mathrm{hz}$ versus music tuned to $440 \mathrm{hz}$ for improving sleep in patients with spinal cord injuries: a double-blind cross-over pilot study. Acta Biomed. 2020;91(12-S):1-15. doi:10.23750/abm.v91i12-S.10755

41. Jespersen KV, Koenig J, Jennum P, Vuust P. Music for insomnia in adults. Cochrane Database Syst Rev. 2015. doi:10.1002/14651858. CD010459.pub2

42. Bang YR, Choi HY, Yoon IY. Minimal Effects of Binaural Auditory Beats for Subclinical Insomnia: a Randomized Double-Blind Controlled Study. J Clin Psychopharmacol. 2019;39(5):499-503. doi:10.1097/JCP.0000000000001097
43. Yamasato A, Kondo M, Hoshino S, et al. How Prescribed Music and Preferred Music Influence Sleep Quality in University Students. Sleep. 2020;45.

44. Loewy J. Music therapy as a potential intervention for sleep improvement. Nat Sci Sleep. 2020;12:1-9. doi:10.2147/NSS.S194938

45. Garcia-Rill E. Reticular Activating System. In: Encyclopedia of Neuroscience. Elsevier Ltd; 2009:137-143. doi:10.1016/B978008045046-9.01767-8

46. Steriade M, McCormick DA, Sejnowski TJ. Thalamocortical oscillations in the sleeping and aroused brain. Science. 1993;262 (5134):679-685. doi:10.1126/science.8235588

47. Campbell K, Muller-Gass A. The extent of processing of near-hearing threshold stimuli during natural sleep. Sleep. 2011;34 (9):1243-1249. doi:10.5665/SLEEP.1248

48. Davy JL, Burgemeister K, Hillman D, Carlile S. A Review of the Potential Impacts of Wind Turbine Noise in the Australian Context. Acoust Aust. 2020;48(2):181-197. doi:10.1007/s40857020-00192-4

49. Bastien LH, Ladouceur C. EEG Characteristics Prior to and Following the Evoked K-Complex. Can J Exp Psychol. 2000;54 (4):255-265. doi:10.1037/h0087345

50. Choi J, Kwon M, Jun SC. A Systematic Review of Closed-Loop Feedback Techniques in Sleep Studies-Related Issues and Future Directions. Sensors. 2020;20(10):2770. doi:10.3390/s20102770

51. Ngo HVV, Claussen JC, Born J, Mölle M. Induction of slow oscillations by rhythmic acoustic stimulation. J Sleep Res. 2013;22 (1):22-31. doi:10.1111/j.1365-2869.2012.01039.x

52. Ngo HVV, Martinetz T, Born J, Mölle M. Auditory Closed-Loop Stimulation of the Sleep Slow Oscillation Enhances Memory. Neuron. 2013;78(3):545-553. doi:10.1016/J.NEURON.2013.0 3.006

53. Ngo HVV, Miedema A, Faude I, Martinetz T, Mölle M, Born J. Driving sleep slow oscillations by auditory closed-loop stimulation -A self-limiting process. J Neurosci. 2015;35(17):6630-6638. doi:10.1523/JNEUROSCI.3133-14.2015

54. Ong JL, Lo JC, Chee NIYN, et al. Effects of phase-locked acoustic stimulation during a nap on EEG spectra and declarative memory consolidation. Sleep Med. 2016;20:88-97. doi:10.1016/j. sleep.2015.10.016

55. Papalambros PA. Acoustic enhancement of sleep: a tool to boost sleep-mediated benefits on memory and autonomic nervous system functions in aging. Dissertation. 2019;80.

56. Choi J, Won K, Jun SC. Acoustic Stimulation Following Sleep Spindle Activity May Enhance Procedural Memory Consolidation During a Nap. IEEE Access. 2019;7:56297-56307. doi:10.1109/ ACCESS.2019.2913457

57. Henin S, Borges H, Shankar A, et al. Closed-loop acoustic stimulation enhances sleep oscillations but not memory performance. eNeuro. 2019;6(6):ENEURO.0306-19.2019. doi:10.1523/ENEU RO.0306-19.2019

58. Besedovsky L, Ngo H-V V, Dimitrov S, Gassenmaier C, Lehmann R, Born J. Auditory closed-loop stimulation of EEG slow oscillations strengthens sleep and signs of its immune-supportive function. Nat Commun. 2017;8(1):1984. doi:10.1038/s41467-017-02170-3

59. Diep C, Garcia-Molina G, Jasko JG, et al. Acoustic enhancement of slow wave sleep on consecutive nights improves alertness and attention in chronically short sleepers. Sleep Med. 2021;81:69-79. doi:10.1016/j.sleep.2021.01.044

60. Debellemaniere E, Chambon S, Pinaud C, et al. Performance of an ambulatory dry-EEG device for auditory closed-loop stimulation of sleep slow oscillations in the home environment. Front Hum Neurosci. 2018;12:88. doi:10.3389/fnhum.2018.00088 
61. Fehér KD, Wunderlin M, Maier JG, et al. Shaping the slow waves of sleep: a systematic and integrative review of sleep slow wave modulation in humans using non-invasive brain stimulation. Sleep Med Rev. 2021;58:101438. doi:10.1016/j.smrv.2021.101438

62. Schneider J, Lewis PA, Koester D, Born J, Ngo H-V V. Susceptibility to auditory closed-loop stimulation of sleep slow oscillations changes with age. Sleep. 2020;43(12):1-10. doi:10.1093/sleep/zsaa111

63. Schade MM, Mathew GM, Roberts DM, Gartenberg D, Buxton O. Enhancing Slow Oscillations and Increasing N3 Sleep Proportion with Supervised, Non-Phase-Locked Pink Noise and Other Non-Standard Auditory Stimulation During NREM Sleep. Nat Sci Sleep. 2020;12:411-429. doi:10.2147/NSS.S243204

64. Héritier H, Vienneau D, Foraster M, et al. Transportation noise exposure and cardiovascular mortality: a nationwide cohort study from Switzerland. European Journal of Epidemiology. 2017;32 (4):307-315. doi:10.1007/s10654-017-0234-2

65. Riedy SM, Smith MG, Rocha S, Basner M. Noise as a sleep aid: a systematic review. Sleep Med Rev. 2021;55:101385. doi:10.1016/j. smrv.2020.101385

66. Beck J, Loretz E, Rasch B. Activating the concept of "relaxation" during sleep using relaxation-related words. Sleep. 2021;44 (Supplement_2):A15-A16. doi:10.1093/sleep/zsab072.034

67. World Health Organization. Environmental Noise Guidelines for the European Region. WHO; 2018. http://www.euro.who.int/pubrequest. Accessed May 11, 2021.

68. Berglund B, Lindvall T, Schwela DH. New Who Guidelines for Community Noise. Noise Vib Worldw. 2000;31(4):24-29. doi:10.1260/0957456001497535

69. Micic G, Zajamsek B, Lack L, et al. A Review of the Potential Impacts of Wind Farm Noise on Sleep. Acoust Aust. 2018;46 (1):87-97. doi:10.1007/s40857-017-0120-9

70. Liebich T, Lack L, Hansen K, et al. A systematic review and meta-analysis of wind turbine noise effects on sleep using validated objective and subjective sleep assessments. J Sleep Res. 2020. doi: $10.1111 /$ jsr. 13228

71. van Kamp I, Simon S, Notley H, Baliatsas C, van Kempen E. Evidence Relating to Environmental Noise Exposure and Annoyance, Sleep Disturbance, Cardio-Vascular and Metabolic Health Outcomes in the Context of IGCB (N): a Scoping Review of New Evidence. Int J Environ Res Public Health. 2020;17(9):3016. doi:10.3390/ijerph17093016
72. Aili K, Åström-paulsson S, Stoetzer U, Svartengren M, Hillert L. Reliability of actigraphy and subjective sleep measurements in adults: the design of sleep assessments. J Clin Sleep Med. 2017;13(1):39-47. doi: $10.5664 /$ jcsm. 6384

73. Trimmel K, Eder HG, Böck M, Stefanic-Kejik A, Klösch G, Seidel S. The (mis)perception of sleep: factors influencing the discrepancy between self-reported and objective sleep parameters. J Clin Sleep Med. 2021;17(5):917-924. doi:10.5664/jcsm.9086

74. Öhström E, Björkman M. Effects of noise-disturbed sleep-A laboratory study on habituation and subjective noise sensitivity. $J$ Sound Vib. 1988;122(2):277-290. doi:10.1016/S0022-460X(88)80354-7

75. Tamura Y, Horiyasu T, Sano Y, et al. Habituation of sleep to a ship's noise as determined by actigraphy and a sleep questionnaire. J Sound Vib. 2002;250(1):107-113. doi:10.1006/jsvi.2001.3900

76. Kuroiwa M, Xin P, Suzuki S, Sasazawa Y, Kawada T, Tamura Y. Habituation of sleep to road traffic noise observed not by polygraphy but by perception. J Sound Vib. 2002;250(1):101-106. doi:10.1006/ jsvi.2001.3901

77. Öhrström E. Sleep disturbance, psycho-social and medical symptoms-A pilot survey among persons exposed to high levels of road traffic noise. J Sound Vib. 1989;133(1):117-128. doi:10.1016/ 0022-460X(89)90986-3

78. Frei P, Mohler E, Röösli M. Effect of nocturnal road traffic noise exposure and annoyance on objective and subjective sleep quality. Int J Hyg Environ Health. 2014;217(2-3):188-195. doi:10.1016/j. ijheh.2013.04.003

79. Leventhal G, Pelmear P, Benton S. A Review of Published Research on Low Frequency Noise and Its Effects. Sleep. 2003.

80. Maes J, Verbraecken J, Willemen M, et al. Sleep misperception, EEG characteristics and Autonomic Nervous System activity in primary insomnia: a retrospective study on polysomnographic data. Int $J$ Psychophysiol. 2014;91(3):163-171. doi:10.1016/j. ijpsycho.2013.10.012

\section{Publish your work in this journal}

Nature and Science of Sleep is an international, peer-reviewed, open access journal covering all aspects of sleep science and sleep medicine, including the neurophysiology and functions of sleep, the genetics of sleep, sleep and society, biological rhythms, dreaming, sleep disorders and therapy, and strategies to optimize healthy sleep.
The manuscript management system is completely online and includes a very quick and fair peer-review system, which is all easy to use. Visit http://www.dovepress.com/testimonials.php to read real quotes from published authors. 